\begin{tabular}{|c|c|}
\hline Title & Phosphorylated chitin increased bone formation when implanted into rat calvaria with the Ti-device \\
\hline Author(s) & $\begin{array}{l}\text { Song, Qin; Y agami, Kimitoshi; Furusawa, T oshitake; Takita, Hiroko; Kurasaki, Masaaki; Tokura, Seiichi; Morimoto, } \\
\text { Koichi; Sammons, Rachel; Iku, Shouhei; Kuboki, Y oshinori }\end{array}$ \\
\hline Citation & $\begin{array}{l}\text { Bio-medical materials and engineering, 31(1), 47-57 } \\
\text { https://doi.org/10.3233/BME-201079 }\end{array}$ \\
\hline Issue Date & $2020-05-12$ \\
\hline Doc URL & http:/hdl.handle.net/2115/79046 \\
\hline Rights & The final publication is available at I OS Press through http://dx.doi.org/10.3233/BME-201079 \\
\hline Type & article (author version) \\
\hline File Information & Biomed. Mater. Eng. 31(1) 47-57.pdf \\
\hline
\end{tabular}

Instructions for use 
To be submitted to Biomedical Materials and Engineering

\title{
Phosphorylated chitin increased bone formation when implanted into rat calvaria with the Ti-device
}

\author{
Qin Song $^{a}$, Kimitoshi Yagami ${ }^{b}$, Toshitake Furusawa ${ }^{c}$, Hiroko Takita ${ }^{\mathrm{d}}$, Masaaki Kurasaki ${ }^{\mathrm{e}}$, \\ Seiichi Tokura ${ }^{\text {e }}$, Koichi Morimoto ${ }^{\text {, }}$, Rachel Sammons ${ }^{\text {g }}$, Souhei Iku ${ }^{\text {h }}$,Yoshinori Kuboki ${ }^{\text {* }}$ \\ 'College of Pharmacy and Bioengineering, Chengdu University, Chengdu, Sichuan 610106, China \\ b \\ bepartment of Oral Health Promotion, Graduate School of Oral Medicine, Matsumoto Dental \\ University, Shojiri, Japan 399-0781 \\ 'Department of Bio-engineering, Graduate School of Science and Engineering, Yamagata \\ University, Tsuruoka, Japan 982-0001 \\ ${ }^{\text {d}}$ Faculty Dental Medicine, Hokkaido University, Sapporo Japan 060-8586 \\ eFaculty of Environmental Earth Science, Hokkaido University, Sapporo Japan 060-0810 \\ fDepartment of Biotechnological Science, Kinki University, Kinogawa, Wakayama, Japan 649-6493 \\ g Department of Biomaterials, School of Dentistry, Birmingham University, UK \\ hJiangsu Alphay Biologeal Technology Co., Ltd, Biotechnology Industrial Park No. 1, Suqian, \\ Jiangsu, 223800 China
}

Key words: Chitin, Phosphorylation, enhanced bone formation, Ti-device

Topics: Phosphorylation, Chitin, Bone formation, Calcification

*Corresponding author: Yoshinori Kukoki, Room A507, Faculty of Environmental Earth Science, Hokkaido University, Sapporo Japan 060-0810, E-mail: yk24936@gmail.com, Tel: 011-706-2252. Fax: 011-706-3864

Abbreviations: SIBLING, small integrin-binding ligand, n-linked glycoprotein; PBS, phosphate buffered saline; I-AC, type I acid soluble collagen; I-PC, type I pepsin soluble collagen 


\section{Abstract}

Swedish orthopedist P. I. Brånemark discovered accidentally a strong physical bond between living bone and titanium in the 1950s. This remarkable phenomenon is now widely applied for all of the dental and orthopedic titanium implants. However, until recently almost nothing is known about the biochemical mechanism concerning this strong bond. We were the first to show that a group of RGD-containing phospho-proteins in bone (SIBLING-family) binds with titanium and increased more than 100 times of bone formation when implanted into bone, therefore these bone proteins play a crucial role in bone formation around the titanium. But for the wider clinical application we need more simpler biomolecules for the modification of Ti surface. In this point, we anticipated that the phosphorylated chitin, that is a derivative of major component of exoskeleton of Crustacea, may be a good candidate for intermediate molecule between $\mathrm{Ti}$ and bone. In this study we demonstrated that phosphorylated chitin induced higher calcification in vitro when it was coated on titanium device and compared with the non-coated devices. Furthermore, we demonstrated that the coated Ti-device induced the higher bone formation than the uncoated control, when implanted into rat bone. These findings suggested that phosphorylated chitin may play a role of partial substitute of SUBLINB protein in bone.

\section{Introduction}

\section{Background}

A remarkable discovery was made in 1950 s by Brånemark (Intro-1) that solid state titanium (Ti) and the living bone tissues were strongly bound together. It was found during his experiments involving direct observation of the blood stream in rabbit bone marrow using a light microscope which was equipped with a titanium objective lens holder (Intro-1). This finding naturally attracted the attentions of orthopedic and dental researchers with expectations of creating new artificial bone and tooth. After more than 60 years, medical applications of titanium for reconstruction in artificial joints and roots of teeth have been universally developed. Until recently, however, it is not really understood mechanism how the bone formation is initiated and how the interface between bone and Ti is maintained.

In 2016, we have found that a group of phosphorylated and RGD-contained proteins (SIBLING family) in bone tissue possessed binding ability with Ti. And moreover, it was found that bone formation was highly promoted when the SIBLING proteins were coated on the Ti device and implanted into animal bone (Intro-2-5). This well explained initial mechanism of 
preferential bone formation on the surface of $\mathrm{Ti}$, and we call the SIBLING proteins in bone as "implant proteins"

\section{Needs for a good substitute}

However, natural SIBLING proteins are not so easy to apply clinically as a coating biomaterial of Ti implants, because the biochemical purification of SIBLING from bone requires complex process, also genetic biosynthesis of them is highly expensive.

Therefore, if there a substitute molecule for SIBLING is found, it will be a great benefit for reconstruction therapy of bone and tooth in which $\mathrm{Ti}$ is used. For this purpose we have decided to create a new synthetic material which fulfils two important characters of SIBLING, multiple phosphate groups and a RDG sequence for instances.

P-chitin is expected to be a new biomaterial for regenerative medicine

Chitin is been known to be insoluble in a common solvent. But we have already found that the phosphorylation of chitin rendered this highly insoluble biomolecule into the solubilized form. This finding was a highly important breakthrough, because the soluble form of chitin can be manipulate with many biochemical processes (Intro-1-5). Furthermore, we discovered that phosphorylated and solubilized chitin bind with titanium which verified by using the chromatography packed with titanium beads (1-5).

In this study, we tried to explore to the functions of phosphorylated chitin (P-chitin). We coated a Ti device of web form (TW) with the purified P-chitin and checked two functions of the P-chitin coated TW. First, checked whether the P-chitin coated TW promote in vitro calcification, when we soak it in the calcification solution. Second, we studied in vivo whether P-chitin coated TW actually promote higher bone formation, when we implanted them into animal bone and compared with the result of non-coated TW. Results showed remarkable increased calcification compared with non-coated TW. Second, we found that P-chitin coated Ti-device more effectively induced bone formation than the non-coated control. The both results indicates that $\mathbf{P}$-chitin coated titanium is potentially a promising biomaterials for regenerative medicine in the field of bone and tooth reconstruction.

\section{Materials and Methods}

\subsection{Coating of phosphorylated chitin on 3D-titanium web device}

Commercial chitin was purchased from Fuji-Film Wako, Tokyo. They were chemically phosphorylated by the methods previously (Intro 2-5). Briefly, phosphorylation of chitin was achieved by phosphorous pentoxide in dimethyl sulfoxide (DMSO) or hexane as a solvent and methane sulfonic acid as a catalyst. Two grams of chitin powder was suspended in $40 \mathrm{ml}$ of DMSO or hexane in which $4.5 \mathrm{~g}$ of phosphorous pentoxide (molar ratio of phosphorous 
pentoxide against acetyl glucosamine residue was about 3:1) and $1.5 \mathrm{ml}$ of methane sulfonic acid were dissolved beforehand. The mixture was stirred for $6 \mathrm{~h}$ at $70 \sim 80^{\circ} \mathrm{C}$. The reaction was stopped by the addition of acetone to obtain final concentration of $60 \mathrm{vol} \%$. The reaction product was collected by filtration and rinsed by acetone several times and air-dried. The dried product was dissolved in $\mathbf{5 0} \mathrm{ml}$ of distilled water followed by dialysis for $48 \mathrm{~h}$ against distilled water in a refrigerator at $10^{\circ} \mathrm{C}$ and lyophilized. This crude phosphorylated chitin was further purified by titanium beads packed column chromatography and the fraction retained in the column (Intro 1-6) was used for the experiments of the calcification in vitro and the enhancement of bone formation in vivo.

Titanium devices in a web form (TW) used in this study was 3D unwoven texture that is composed of thin titanium fibers of $80 \mu \mathrm{m}$, originally developed in this laboratory (Method 6-10) and available in various sizes (Zellez ${ }^{\circledR}$, Hi-Lex corporation-Funakoshi, Japan). This material has been already verified to be one of ideal 3D titanium scaffold for bone ingrowth and formation (Method 6-10). The TW used in this study were the disc-forms of 13 x 1.5 and 5 $x 1.5 \mathrm{~mm}$ for the calcification experiment and those of $5 \times 1.5 \mathrm{~mm}$ for the bone formation experiment, both with $87 \%$ porosity. In order to combine the TW and the purified p-chitin, the 20 pieces of the TW were immersed in $2 \mathrm{ml}$ of the $0.2 \%$ solution of the purified p-chitins and incubated at $15^{\circ} \mathrm{C}$ for $15 \mathrm{~min}$. After immersion, p-chitin coated TW were air-dried in a clean bench and stored at 5 .

\subsection{In Vitro calcification of the p-chitin coated TW}

Calcifiability of p-chitin coated TW was tested by the incubation method in the calcifying solution with high $\mathrm{Ca}$ and phosphate ions concentration to obtain hydroxyapatite, which have reported previously (Method 10 and 11). Briefly, this method includes the incubation in the calcification solution which include $15 \mathrm{mM}$ Ca ion and $9 \mathrm{mM}$ phosphate ion which are 6 times higher than the physiological fluid. This solution has been verified to induce hydroxyapatite rapidly under the described conditions (Method 10 and 11).

Pieces of p-chitin coated TW (5 x $1.5 \mathrm{~mm})$ were immersed in the calcifying solution, whose contents were $15 \mathrm{mM} \mathrm{CaCl} 2,9 \mathrm{mM} \mathrm{K2} 2 \mathrm{PO} 4,0.7 \mathrm{M} \mathrm{NaCl}$ and $0.02 \mathrm{M} \mathrm{NaHCO}$, $\mathrm{pH}$ of which was adjusted to 6.01 by the passage of gaseous carbon dioxide. The bottles that contained the samples were tightly sealed and incubated for $48 \mathrm{hr}$ at $37{ }^{\circ} \mathrm{C}$. By the end of this period the coated samples were rinsed with demineralized water, lyophilized and weighed. These samples were then immersed again in fresh calcifying solution as described above. The incubation was 
continued for 20 days with change of the solution every other day, after which these samples were rinsed and lyophilized as above (Method 10-11).

\section{Method-Ref 10}

Yoshinori Kuboki, Shouhei Iku, Ryota Yoshimoto, Tohru Kaku, Hiroko Takita, Dong Li, Yasuo Kokai, Shunji Yunoki, and Rachel L. Sammons, Kazuhide Ozeki, and Teruo Miyata. Calcified Honeycomb-shaped Collagen Maintains its Geometry in Vivo and Effectively

Induces Vasculature and Osteogenesis Nano Biomedicine 2009 1(2), 85-94, 2009

\section{Method-Ref 11}

Nucleation of biomimetic Ca-P coatings on ti6A14V from a SBF x 5 solution: influence of magnesium.

Barrere F, van BC, de GK, Layrolle P.

Biomaterials. 2002 May;23(10):2211-20.

\subsection{Implantation into rat calvaria}

Eight-weeks-old male rats (Wistar, 200-230 g) were anesthetized with pentobarbital sodium (3.6 mg/100 g body weight, Nembutal R, Dainippon Sumitomo Pharma, Japan). The TW/TiBP composites were implanted into the holes with diameter of $5 \mathrm{~mm}$ created by diamond bur and trepan bur. After implantation, periostea and skin were carefully placed back and sutured. The protocol of the animal experiments was approved by the Committee for Animal Experiments in Kanagawa Dental College. It was carried out in accordance with guidelines proposed by the Institutional Animal Care and Use Committee. One piece of either the TW/TiBP composite or TW alone was implanted into one rat calvaria. After one month, the samples were removed for examination as described previously (4). Parts of samples were retrieved after one week to see early responses.

\subsection{Histological observation}

Excised pieces of cranial bone, which contained implant, were fixed in $10 \%$ neutral formaldehyde and embedded in polyester resin (Technovit 8100 Heraeus Kulzer, Germany) to prepare undecalcified sections according to the manufacturer's protocol. Three- $\mu$ m-thick of three consecutive sections were obtained from each specimen using an Exakt grinding machine assisted with mechanical digital micrometer (Exakt, Norderstedt, Germany). Sections were stained with Villanueva osteochrome bone stain (Polysciences, Inc., Warrington, PA, USA). 


\subsection{Statistical Analysis}

New bone areas within the p-chitin coated and uncoated TWs which were implanted into rat calvaria were analyzed by Image-J and compared. Ratios of the new bone areas in each groups (7 histological samples from each group) to the average value of new bone areas in the uncoated TW group at 2 weeks were calculated. Means, standard deviation and statistical significance by Student's t-test were and presented.

\section{Results}

\subsection{Enhancing calcification on the titanium device.}

Figure 1 showed the scanning electron micrographs with different magnifications of the intact TW (A, C, E and G) and the p-chitin coated and calcified TW after incubation in the calcifying solution for 20 days (B, D, F and H).

At lower magnification, crystals of precipitated materials seemed to be flat or irregular half -bulbar shapes which are attached on the surface of TW (Fig. 1D). But in the higher magnifications (Fig. 1F and $\mathrm{H}$ ) these structures are found to be composed of assembled leaves or plates shapes which were grown from the surface of TW.

Figure 2 summarize the increase amount of the precipitation in the p-chitin coated TW, compared with non-coated TW. Remarkable increases were observed on the weights in the coated TW, with 6.7, 4.8 and 4.9 times higher than the those of the uncoated TW at 6, 12 and 20 days of incubation, respectively. 


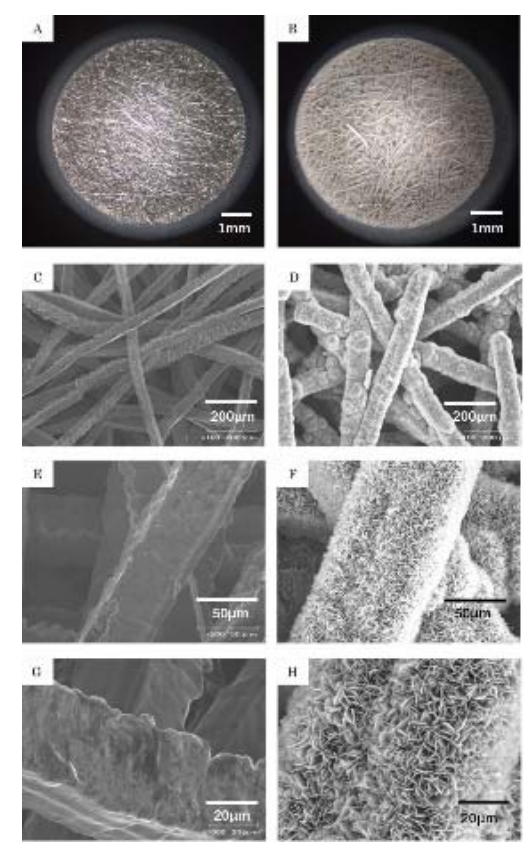

Figure 1 legend

Scanning electron micrographs of the untreated original TW (5 x $1.5 \mathrm{~mm})(\mathrm{A}, \mathrm{C}, \mathrm{E}$ and $\mathrm{G})$ and the same TW which was coated with p-chitin and incubated in the calcifying solution (Method-ref 10) for 20 days (B, D, F and H).

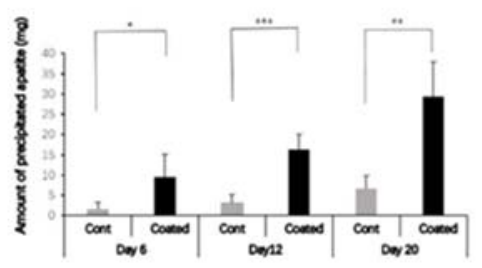

Figure 2 legend

Ability of enhancing calcification on the p-chitin coated TW

The p-chitin coated TW (19 $\times 1.5 \mathrm{~mm})$ were incubated in the calcification solution for 6,12 and 20 days, and increases of the weights $(\mathrm{mg})$ were expressed as the ratios to the average weight of the uncoated TW without incubation, which are 6.7, 4.8 and 4.9, respectively. Student' $P$ values were $0.0359 *(<0.05), \quad 0.00587 * *(<0.01)$ and $0.00267 * * *(<0.005)$ between the average values of uncoated control and coated groups, at day 6,12 and 20 , respectively. 
3.2 Enhancement of bone formation in the P-chitin-coated TW compared with TW alone

Figures 3A-D showed the histological observation of the p-chitin coated TW and TW alone as control which were implanted into rat calvaria and retrieved 2 weeks after implantation. It can be seen that implantation of p-chitin coated TW (Fig. 3A and B) induced active new bone formation within the framework of TW. In contrast, TW alone induced scarcely new bone formation (Fig. 3C and D). There was some heterogeneity in the bone formation within the TW. Where the density of Ti fibers (black lines) were high in the middle part, bone formation was active. In the higher magnification (Fig. 3D), many capillaries are observed (indicated by white arrows).

These enhancements of bone formation by the p-chitin coated TW were still observed in 4 weeks after implantation. Figures 4A-D showed the histological observation of the p-chitin coated TW and TW alone as control implanted into rat calvaria and retrieved 4 weeks after implantation.

Within the each tissue section of the retrieved TW, the ratio of area occupied bone to the total area of TW were analyzed and compared between the ratios of noncoated and p-chitin coated TW. Figure 5 demonstrates the bone occupancy in each section were compared against the bone occupancy ratio of the uncoated TW at 2 weeks, which was the lowest. At 2 weeks, bone occupancies of the chitin coated TW was 4 times at 2 weeks, and 1.6 times higher at 4 weeks than those of the uncoated TW. 
Figure 3

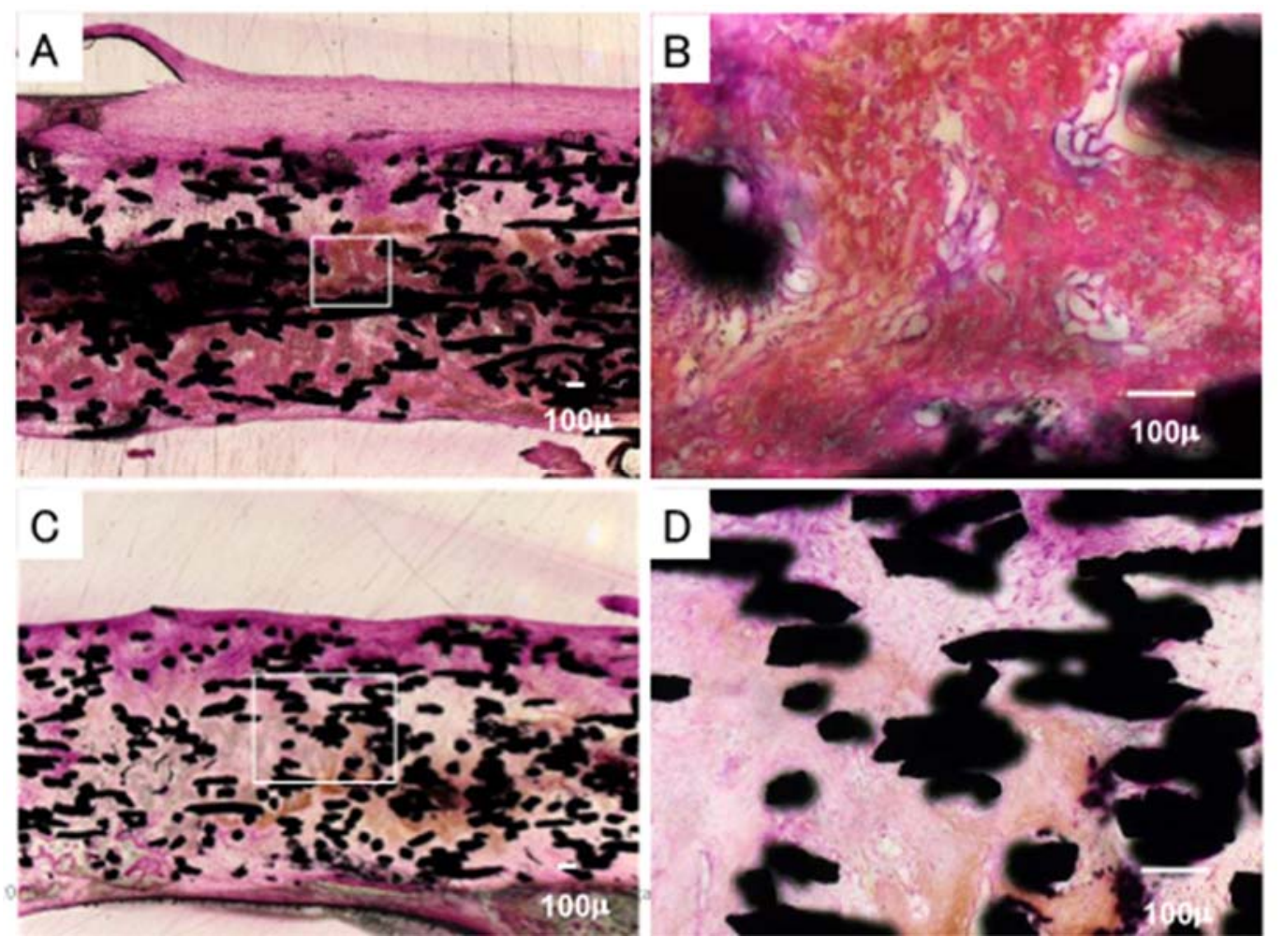


Figure 4

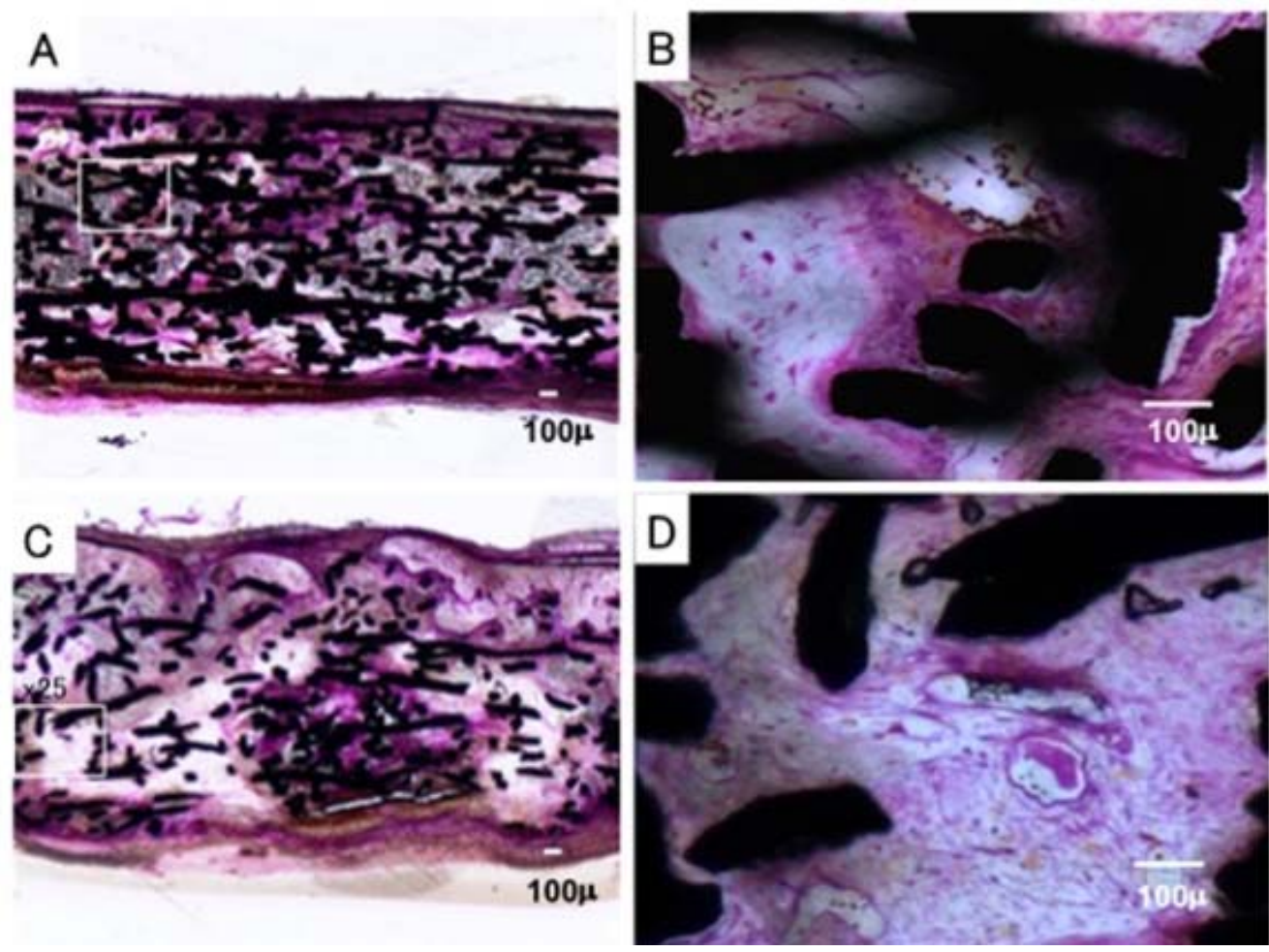

Figure 3 legend

Histological observation of P-coated (A and B) and non-coated (C and D) Ti-devices, TW implanted into rat calvaria for 2 weeks

$B$ is the enlarged picture of the area defined by rectangle in $A$, and $D$ is the same in $C$.

Figure 4 legend

Histological observation of P-coated (A and B) and non-coated (C and D) Ti-devices, TW implanted into rat calvaria for 4 weeks

$B$ is the enlarged picture of the area defined by rectangle in $A$, and $D$ is th 


\section{Figure 5}

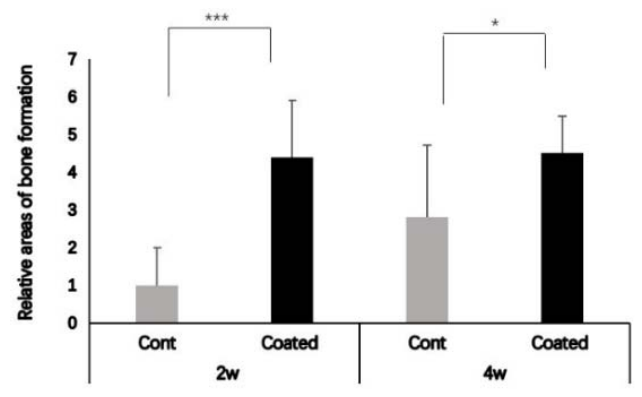

Figure 5 Legend

Enhancement of bone formation in the p-chitin coated TW compared with those of the control (noncoated) TW when they were implanted into rat bone for 2 an 4 weeks

In each tissue section of the retrieved TW, the ratio of area occupied bone to the total area of TW were analyzed and compared. Bone occupancy in each section were compared against the bone occupancy ratio of the uncoated TW at 2 weeks, which was the lowest. At 2 weeks, bone occupancies of the chitin coated TW was 4 times at 2 weeks, and 1.5 times higher at 4 weeks than those of the uncoated TW $(n=6)$.

\section{Discussion}

Increased calcifiability of P-chitin-coated TW compared with TW and it's mechanism As shown in Fig. 1, increased effect on the calcification of p-chitin coating on TW was remarkable. This effect was easily understandable considering that every $\mathrm{C6}$ hydroxy groups of N-acetyl glucosamine residue of this p-chitin have phosphorylated (1-5). Presence of numerous phosphate groups with regular arrangement on immobilized linear high molecular substance is one of the ideal substrates for apatite formation (Discuss-ref 301-305). In the liner molecule such as chitin, phosphate groups bound to $\mathrm{C6}$ are lined up straightly with the distance of approximately $1 \mathrm{~nm}$ (Discuss-ref 301-305). 
On the bone-enhancing ability of p-chitin coated TW

Figure 3 and 4 showed the clear enhancement of bone formation

Mechanism of bone enhancement of bone

There are two explanations for the increased bone formation in the p-chintin coated TW.

1) Phosphate groups in the p-chitin promoted local calcification on the surface of TW, which obviously leads apatite formation, on which osteoblasts more easily adhered and started more rapidly bone formation than the naked surface of TW.

2) Possibility of P-chitin itself has presently unknown favorable effects for adhesion, growth and differentiation of osteoblasts.

\section{General conclusion}

Strong bond between the living bone and Ti which discovered by Branemark is highly valuable for regenerative medicine for bone and tooth reconstruction. But there has been one important short coming: it takes a considerably long time of months to acquire a practical bond strength. This is reasonable since this bond is based upon biological process of bone formation around the surface of Ti, not based on the simple chemical reaction. Numerous efforts have been made to accelerate fixation of Ti implant into bone, which include giving roughness to the Ti surface, 3D geometrical structures (Discussion ref 41-45), chemical modification of the Ti surface and coating the surface with various substances such as hydroxyapatite. We are the first to report that P-chitin coating is highly effective to enhance bone formation. Although the detailed mechanism for enhancement of bone formation is still to be elucidated, we conclude that p-chitin is highly active biomaterial for calcification and bone formation when combined with Ti devise. 